\title{
Prevalence of myocardial viability in dysfunctional areas by cardiovascular magnetic resonance in patients with coronary artery disease
}

\author{
Lara Bakhos*, Maria Manuela Izquierdo-Gomez, Edwin Wu, Mihai Gheorghiade, Jeffrey J Goldeberger, \\ Alan H Kadish, Daniel C Lee
}

From 2011 SCMR/Euro CMR Joint Scientific Sessions

Nice, France. 3-6 February 2011

\section{Background}

The presence of dysfunctional yet viable myocardium (DV-Myo) is known to predict functional recovery after revascularization and long-term prognosis. We sought to define the prevalence of DV-Myo by cardiovascular magnetic resonance imaging (CMR) in patients with coronary artery disease.

\section{Methods}

We analyzed 181 patients from the DEfibrillators To REduce Risk by MagnetIc ResoNance Imaging Evaluation Trial. All patients underwent cine and contrastenhanced (CE) CMR. Cine and CE studies were scored on a 17-segment model by the consensus of two readers. Cine images were scored for wall motion (WM): $0=$ normal, 1 = mild hypokinesis, 2 = moderate to severe hypokinesis, 3 = akinesis, 4 = dyskinesis. CE images were scored for hyperenhanced (HE) infarct transmurality: 0 = none, $1=1-25 \% \mathrm{HE}, 2=26-50 \% \mathrm{HE}, 3=51-75 \% \mathrm{HE}$, $4=76-100 \%$ HE. Manually planimetered, quantitative analysis was also performed to obtain LV ejection fraction (EF) and infarct size using QMass MR 7.2 (Medis, Leiden, the Netherlands). DV-Myo segments were defined as having WM score $\geq 2$ and HE score $\leq 1$. DVMyo involving 2-4 of 17 segments (12-24\% of LV) was considered prognostically significant only. DV-Myo involving $\geq 5$ of 17 segments ( $\geq 29 \%$ of LV) was considered functionally significant.

\section{Results}

Baseline characteristics included male sex $(84 \%)$ and mean age $61.5 \pm 11.2$ (31-88). Patients were on standard medical therapy, including beta-blockers (93\%), ACE inhibitor/ARB (82\%), anti-platelet (100\%) and lipid lowering agents (94\%). The population had a mean EF of $38.9 \pm 11.6 \%$ (range $11.4-69.1 \%$ ) and infarct size $17.3 \pm$ $10.3 \%$ of LV (range $0-59.5 \%$ ). A total of 3077 segments were evaluated, of which 442 (14\%) segments met criteria for DV-Myo. Prognostically significant DV-Myo was present in $49 / 181$ patients (27.1\%). These patients had a mean of $2.7 \pm 0.7$ affected segments and a mean EF of $34.4 \pm 7.7 \%$. Functionally significant DV-Myo was present in an additional 33/181 patients (18.2\%). These patients had a mean of $8.1 \pm 3.0$ affected segments and a mean EF of $26.2 \pm 8.5 \%$.

\section{Conclusions}

A substantial proportion of patients with CAD have prognostically significant or functionally significant areas of DV-Myo. This highlights the need for viability assessment to guide appropriate therapy in these patients.

Published: 2 February 2011

doi:10.1186/1532-429X-13-S1-P159

Cite this article as: Bakhos et al:: Prevalence of myocardial viability in dysfunctional areas by cardiovascular magnetic resonance in patients with coronary artery disease. Journal of Cardiovascular Magnetic

Resonance 2011 13(Suppl 1):P159. 